\title{
Targeting deubiquitinase USP28 for cancer therapy
}

\author{
Xiaofang Wang ${ }^{1,2}$, Zhiyi Liư ${ }^{3}$, Li Zhang ${ }^{1,2}$, Zhaozhi Yang ${ }^{1,2}$, Xingxing Chen ${ }^{1,2}$, Jurui Luo ${ }^{1,2}$, Zhirui Zhou ${ }^{1,2}$, Xin Mei ${ }^{1,2}$, \\ Xiaoli Yu ${ }^{1,2}$, Zhimin Shao ${ }^{4}$, Yan Feng ${ }^{1,2}$, Shen Fu ${ }^{1,2}$, Zhen Zhang ${ }^{1,2}$, Dongping Wei ${ }^{5}$, Lijun Jia ${ }^{6}$, Jinli Ma ${ }^{1,2}$ and \\ Xiaomao Guo ${ }^{1,2}$
}

\begin{abstract}
As one of the most important post-translational modifications, ubiquitination plays versatile roles in cancer-related pathways, and is involved in protein metabolism, cell-cycle progression, apoptosis, and transcription. Counteracting the activities of the E3 ligases, the deubiquitylating enzymes have been suggested as another important mechanism to modulate the ubiquitination process, and are implicated in cancer as well. In this article, we review the emerging roles of USP28 in cancer pathways as revealed by recent studies. We discuss the major mechanisms by which USP28 is involved in the cancer-related pathways, whereby USP28 regulates physiological homeostasis of ubiquitination process, DNA-damage response, and cell cycle during genotoxic stress. We further review the studies where USP28 was targeted for treating multiples cancers including non-small cell lung cancer, breast cancer, intestinal cancers, gliomas, and bladder cancer. As a result, the clinical significance of targeting USP28 for cancer therapy merits further exploration and demonstration.
\end{abstract}

\section{Facts}

- As one of the most prevalent and important posttranslational modifications, ubiquitination is involved in multiple cancer-related pathways, including cell-cycle progression, apoptosis, receptor downregulation, and gene transcription.

- Antagonizing the activities of E3 ubiquitin ligases, the deubiquitylating enzymes (DUBs) are engaged in cancer-related pathways by modulating the ubiquitination process.

- The general mode of actions for DUBs in cancerrelated pathways are exemplified by their regulation on the stability of oncoproteins or tumor suppressors.

- DUBs have become one of the important cellular targets for cancer treatment.

\section{Open questions}

- Owing to the versatility of USP28-mediated regulation in the cell, what is the dominant mechanism imposed by USP28 for a given cancer type?

- In addition to developing pharmacological inhibitors of USP28, what are other efficient strategies of targeting USP28 that have clinical adaptability and feasibility for treating cancers?

\section{Introduction}

Like phosphorylation, ubiquitination is one of the most prevalent and important post-translational modifications found in the cell, and has multi-faceted roles in normal and pathological cellular processes. In this type of modification, a ubiquitin is attached to the target protein

Correspondence: Jinli Ma (jinlima_c@yahoo.com) or

Xiaomao Guo (guoxm1800@163.com)

${ }^{1}$ Department of Radiation Oncology, Fudan University Shanghai Cancer Center, Shanghai 200032, China

2Department of Oncology, Shanghai Medical College, Fudan University, Shanghai 200032, China

Full list of author information is available at the end of the article Edited by M. Piacentini 


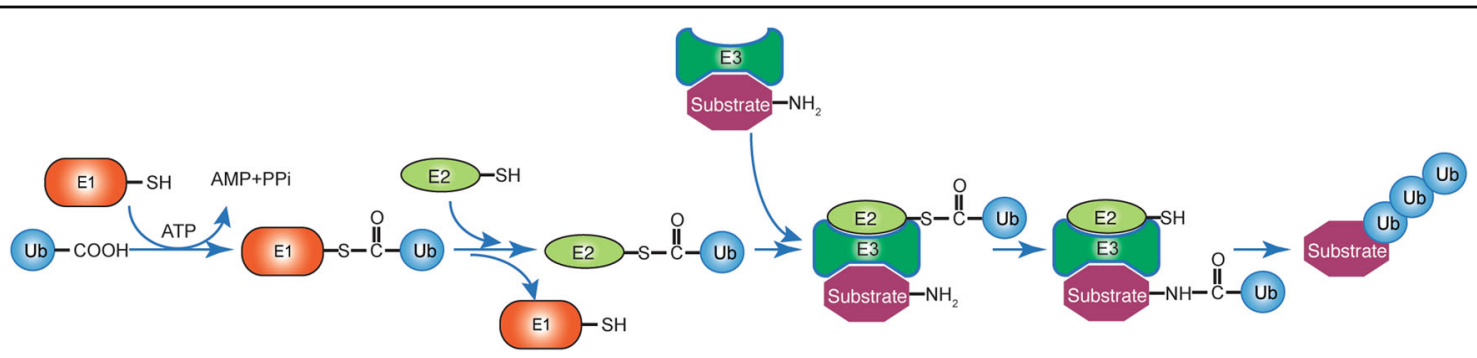

Fig. 1 The ubiquitination process. The ubiquitination process is catalyzed by three types of enzymes that function sequentially. The ubiquitinactivating enzyme, E1, promotes the formation of the thioester bond between the C-terminal carboxyl group of ubiquitin and the E1 cysteine sulfhydryl group. This step is dependent on ATP. In the second step, ubiquitin is transferred from E1 to the active site of the conjugating enzyme, E2. In the last step, the E3 ubiquitin ligase catalyzes the attachment of ubiquitin to the substrate through an isopeptide bond between a lysine of the target protein and the glycine of ubiquitin. The E3 enzymes recognize and discriminate various substrates in the cell, thus determining the substrate specificity in a given ubiquitination process. An E3 enzyme typically carries either one of the two domain structures: the homologous to the E6-AP carboxyl terminus (HECT) domain or the really interesting new gene (RING) domain. Depending on the domain it carries, the catalytic mechanisms may differ.

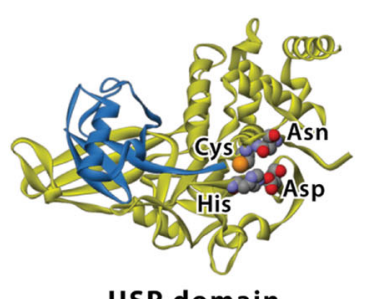

USP domain

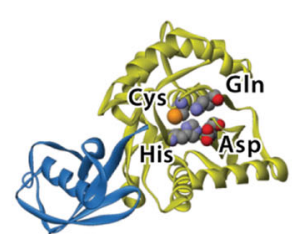

UCH domain

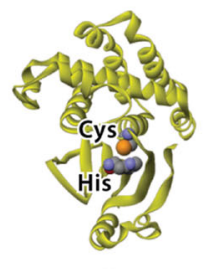

OTU domain

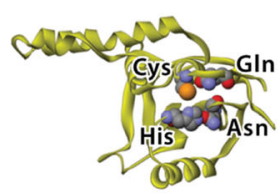

MJD domain

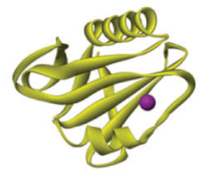

JAMM domain

Fig. 2 Catalytic domain structures of the five subfamilies of deubiquitinases. The catalytic domain structures of the five subfamilies of deubiquitinases vary significantly. USP, UCH, and MJD catalytic domains share conserved residues around the catalytic core, while OTU domain lacks some of them, and these conserved residues are completely missed in the JAMM domain. (Carbon, gray; nitrogen, blue; oxygen, red; sulfur, orange; zinc, purple. Blue ribbon structures represent the complexed ubiquitin. Reproduced with permission from reference ${ }^{3}$.)

through a covalent isopeptidic bond between the Cterminus of the ubiquitin and a lysine residue of the target protein. Implications of ubiquitination in cancer-related pathways have been unveiled in recent years, and they are mostly related to cell-cycle progression, apoptosis, receptor downregulation, and gene transcription. Three types of enzymes function sequentially to add the ubiquitin to the substrate: the ubiquitin-activating enzyme, E1, catalyzes the formation of a thioester bond on ubiquitin. The E2 ubiquitin-conjugating enzyme carries the ubiquitin. Finally, the E3 ligases attach the ubiquitin from E2 to the substrates (Fig. 1). Ubiquitination was first reported as a mechanism to mark proteins for proteasome-mediated degradation. Later, more functions of ubiquitination have been revealed, spanning from modulating protein trafficking to regulating activities of enzymes ${ }^{1}$.

It has been appreciated that the E3 ubiquitin ligases are central to the ubiquitin-conjugation system since they directly interact with substrates and determine the substrate specificity. Some E3 ligases have been implicated in cancer development because they may dictate degradation of oncoproteins or tumor suppressors. For example, agonists of FBW7 can promote degradation of c-MYC and cyclin $\mathrm{E}$ in the tumor cells ${ }^{2}$.

Interestingly, acting reversely by removing the tagged ubiquitin from the substrate protein, deubiquitylating enzymes (DUBs) are implicated as an important mechanism to modulate the ubiquitination process as well, and have been related to cancer, too. There are about 100 DUBs in human, which can be categorized into five families, including ubiquitin-specific proteases (USP), ubiquitin carboxy-terminal hydrolases $(\mathrm{UCH})$, ovarian tumor-like proteases (OTU), metalloproteases, and Machado-Jakob-disease proteases. Except for the DUBs of the JAMM/MPN family, which are metalloproteases, all the other DUBs are cysteine proteases (Fig. 2) ${ }^{3}$. Much knowledge has been accumulated for the functions of DUBs, which are not just limited to reversing ubiquitination of the proteins tagged for proteasomal degradation. It is now known that the functions of DUBs are multidimensional, and encompass apoptosis, protein trafficking, cell cycle regulation, DNA damage repair, chromatin remodeling, and modulating signaling pathways ${ }^{4,5}$. Aside 
from acting on the substrates targeted for degradation, DUBs can also modulate the activities of proteins, therefore affecting the activities of signaling pathways. For instance, in the nuclear factor- $\mathrm{kB}(\mathrm{NF}-\mathrm{kB})$ pathway, the cylindromatosis-associated DUB, CYLD, negatively regulates NF- $\mathrm{KB}$ activation by antagonizing the activity of the E3 ligase tumor necrosis factor receptor-associated factor $2^{6-8}$. The roles of various DUBs in cancer-associated pathways have been reviewed in ${ }^{5}$. In this review, we focus on discussing the specific roles of USP28 in cancer pathways, and their potential therapeutic values in cancer treatment.

\section{The roles of USP28 in cancer-related pathways}

USP28 was initially identified through homology search for USP25. Like USP25, the USP28 gene contains alternatively spliced exons which can produce tissue-specific isoforms of the enzymes ${ }^{9}$. Crystal structures of several USP family enzymes have been resolved, including USP2, USP7, USP8, USP14, and USP2 $1^{10-15}$. These structural studies suggest that USPs possessing a homologous catalytic site are conferred with a similar ubiquitinrecognition mechanism. Therefore, it is likely that all USP family DUBs may share the same mechanism to recognize ubiquitin. USP28 is highly homologous to USP25, both of which contain ubiquitin-associated domain and ubiquitin-interacting motifs in the $\mathrm{N}$ terminal region ${ }^{5}$. The emerging roles of USP28 in cancer pathways have been revealed by some recent studies. Below, we will review the mechanisms accounting for the involvement of USP28 in these pathways (Fig 3, Table 1), hence demonstrating its significance as a therapeutic target for cancer treatment.

\section{Disrupting physiological homeostasis of ubiquitination process}

USP28 was the first FBW7-antagonizing DUB in an shRNA screen using c-MYC stability as a readout in human tumor cells ${ }^{16}$. USP28 can counteract the activity of FBW7 and promote c-MYC stability in cancer cells. Overexpression of USP28 inhibited FBW7-depedent ubiquitination of c-MYC and cyclin E1, both of which are oncoproteins. Depletion of USP28 in some cancer cell lines phenocopies the effect of MYC depletion. As a result, USP28 is required for c-MYC-mediated tumor cell proliferation. FBW7 is an F-box protein and an important component of the SKP1-CUL1-F-BOX (SCF)-type E3 ubiquitin ligase, and determines the substrate specificity of the SCF complex. FBW7 is considered as a tumor suppressor that controls cell proliferation, differentiation, and apoptosis by targeting key transcriptional factors to ubiquitin-directed proteasome degradation ${ }^{17}$. Its functions can be counteracted by DUBs, where Lys48-linked ubiquitin chain can be removed by DUBs before the proteolysis of the tagged protein. The functions of DUBs may cover a wide range of physiological processes in the cells; but the most prominent scenario can be exemplified by the promotion of oncoprotein stability. Pathological dysregulation of the deubiquitination process would lead to deleterious effects, causing the cancer-driving oncoproteins to be accumulated.

In addition to stabilizing the FBW7-substrates, USP28 was also found to directly stabilize FBW7 itself, which leads to reduction of FBW7 substrate proteins. As a result, in contrast to the oncogenic effect of overexpressing USP28 in colorectal cancer ${ }^{18}$, inhibition or deletion of USP28 would otherwise have tumor-promoting effect by destabilizing $\mathrm{FBW} 7^{19}$. This raises an interesting question regarding the dual functions of USP28: whether it is oncogenic or tumor-suppressive? An accountable explanation is that, the levels of USP28 vary considerably in different cells, which can influence the stability and regulation of FBW7 in these cells. As a result, the dual regulation of USP28 on FBW7 may serve as a mechanism to maintain the physiological levels of proto-oncogenic FBW7 substrates. In this model, USP28 may serve as either a tumor promotor or suppressor depending on the status of autocatalytic ubiquitination of FBW7. In the intestine, USP28 deletion does not affect FBW7 stability and attenuates tumorigenesis, while in tissues where FBW7 autocatalytic turnover is favored, deletion of USP28 resembles FBW7 loss-of-function, thus exhibiting a tumor-promoting effect ${ }^{20}$. It is also worth noting that, in addition to the aforementioned oncogenic proteins, other substrates that are known not be associated with FBW7, are also subject to deubiquitination and stabilization by USP28, including Claspin, CHK2, and LSD1 ${ }^{21-23}$. This fact adds another layer of complexity regarding the roles of USP28 in cancer-related pathways.

In addition to affecting the turnover of critical oncoproteins like MYC, USP28 may regulate other essential pathways in cancer progression. A study has proposed a novel mechanism by which USP28 upregulates angiogenesis by antagonizing GSK-3 $\beta$ (glycogen synthase kinase-3 $\beta$ ) and FBW7-dependent degradation of HIF- $1 \alpha$ (hypoxia-inducible factor-1 $\alpha$ ), a major regulator of angiogenesis, carcinogenesis, and various processes by which cells adapt to hypoxic conditions. HIF- $1 \alpha$ is degraded after phosphorylation by GSK-3 $\beta$ and recruitment of FBW7, a process that can be reversed by overexpressing USP28. Importantly, stabilization of HIF-1 $\alpha$ by USP28 is dependent on FBW7, and as a result, FBW7 and USP28 reciprocally regulate angiogenesis and metastasis in an HIF-1 $\alpha$-dependent manner ${ }^{24}$. Therefore, modulation of HIF-1 functions by GSK-3/FBW7/USP28 may contribute to a novel adaptive mechanism for the cell to respond to various signals that affect cell proliferation, differentiation, and apoptosis. The finding offers an 


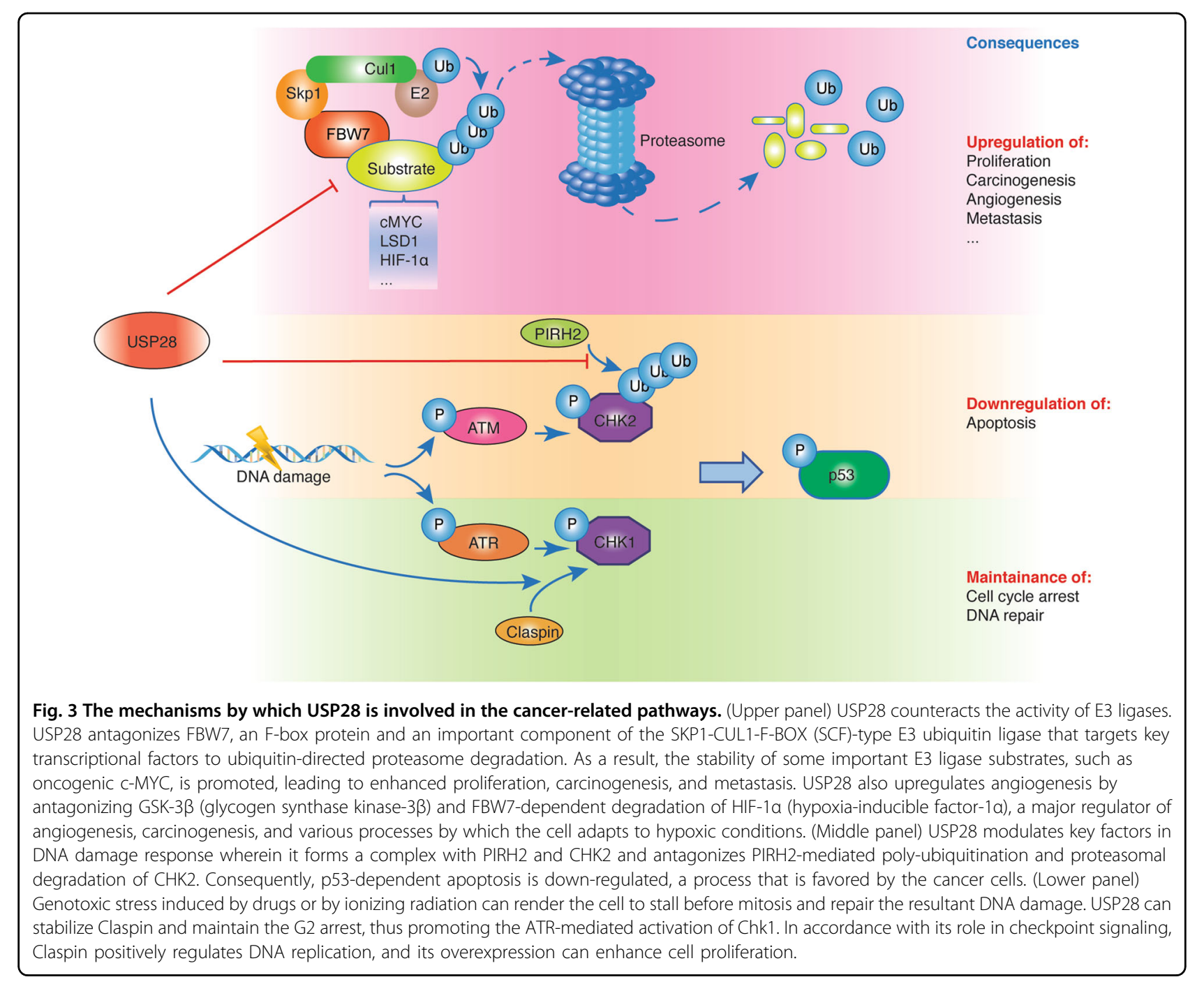

alternative way to regulate HIF-1-dependent cancer pathways by targeting USP28.

\section{Tuning DNA-damage response (DDR)}

In cancer treatment, radiotherapy complements surgery and chemotherapy. Despite the developments in planning and application of radiotherapy that result in improved cure rate, challenges of radioresistance and radiationinduced damage persist to impair the success of radiotherapy $^{25-27}$. The major effect of radiotherapy is the ionizing damages caused to the DNA, where base damage, single strand break (SSB), and double strand break (DSB) can occur. The cell has exquisite mechanisms to sense and repair the DNA damages. But if these pathways are disturbed and dysregulated, as observed in the tumor cells, radioresistance can develop and nullify the therapeutic efforts. An early study has shown USP28 plays an important role in the control of the $\mathrm{DDR}^{22}$. The DDR signals are sensed and initiated by phosphatidylinositol 3-kinase-like kinases (PIKKs), such as ATM and ATR, transduced by various cellular factors, before activating their downstream checkpoint kinases like CHK1 and CHK2. PIKK specificity factors recruit PIKKs to different forms of DNA damage (i.e., base damage, SSB, and DSB) and regulate their activities. Checkpoint mediators, including 53BP1, serve as scaffolds for PIKK complexes and their substrates. Theses checkpoint mediators are critical in controlling the response to irradiation (IR)induced DDR ${ }^{28}$. USP28 was initially identified as a 53BP1binding partner in a tandem affinity purification experiment. Mechanistically, USP28 deubiquitinates and stabilizes the specificity factors and mediators of ATM and ATR signaling by protecting them from ubiquitinationmediated proteasome degradation. The regulation on these factors and mediators, including 53BP1, Claspin, and MDC1, is dependent on the physical association with USP28. The catalytic domain of USP28 is required for this regulation as well, and a dominant-negative mutant of a 


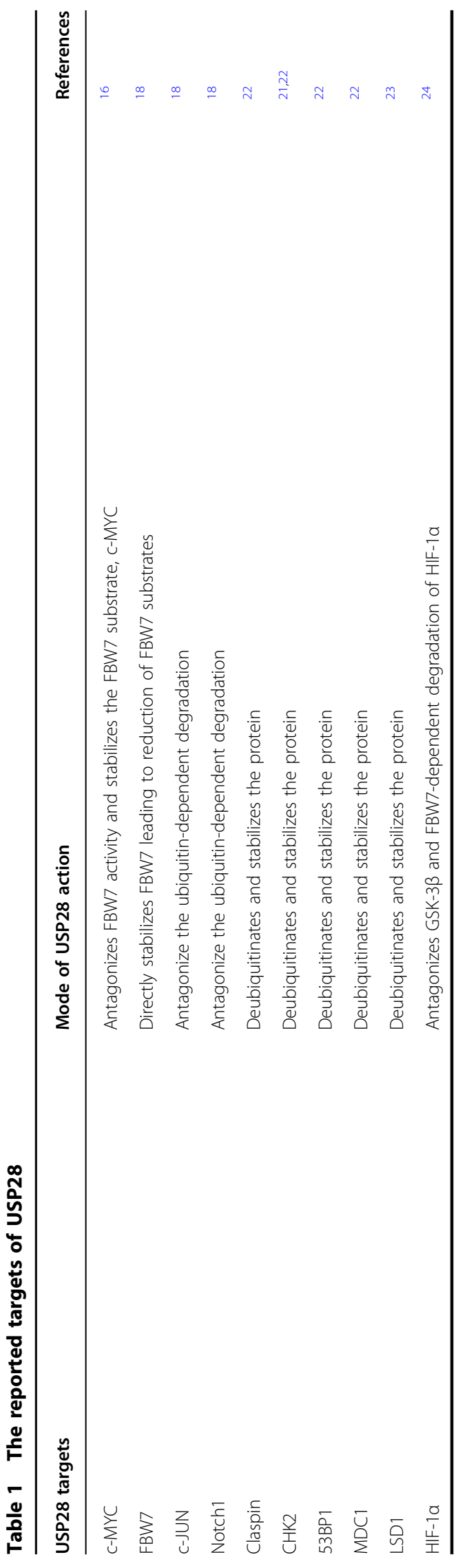

catalytic cysteine phenocopies USP28 knockdown cells. Therefore, USP28 may restrain ubiquitination-mediated proteolysis of the components in DDR. Interestingly, in another study, CHK2 is shown to be directly regulated by USP28. The ubiquitin E3 ligase PIRH2 (p53-induced protein with a RING-H2 domain) interacts with $\mathrm{CHK} 2$ and mediates its ubiquitination-directed proteasomal degradation. USP28 forms a complex with PIRH2 and $\mathrm{CHK} 2$ and counteracts the negative regulation of PIRH2 on $\mathrm{CHK} 2^{21}$. Although in this system, the phenotype of USP28-deficient cells is unclear, CHK2 is expected to be destabilized by knockdown of USP28, which may lead to the negative regulation of DDR. However, it is worth noting that regulation of DDR by USP28 has another layer of complexity-USP28 is involved in multiple pathways that can have opposing functions. It can either promote apoptotic death by stabilizing factors of the CHK2-p53 pathway, or promote IR resistance by regulating Clas$\operatorname{pin}^{22,29}$. Therefore, the effect of USP28 on survival during DDR is determined by the net balance of these opposing pathways in the cell under the physiological milieus, and can be tissue-and cell type-dependent.

\section{Maintaining G2 arrest during genotoxic stress}

During genotoxic stress induced either by drugs or by IR, the cell has to repair the resultant DNA damage before progressing to mitosis. USP28 has been shown to maintain the G2 arrest by stabilizing Claspin, a key regulator of $\mathrm{CHK}^{30}{ }^{30}$. Claspin can stimulate ATR-dependent CHK1 activation after genotoxic stress. After the stress is relieved during G2 phase when the cell is recovered from DNA replication stress, Claspin is ubiquitinated and degraded via SCF ${ }^{\beta T r c p}$. Therefore, degradation of Claspin serves as a mechanism for the cell to shut off the checkpoint and enter the $M$ phase during the cell cycle. Interestingly, Claspin level oscillates between the previous and next rounds of G1/S transition, and thus is subject to active degradation $^{31-34}$. Proteolysis of Claspin in G1 phase is required for the cell to maintain the G0/G1 state and prevent premature entry into $S$ phase. Also, in parallel with its role in checkpoint signaling, Claspin positively regulates DNA replication-overexpression of Claspin can enhance both tumor and normal cell proliferation ${ }^{29}$. As a result, it is likely that stabilization of Claspin by USP28 may have further implications in cell cycle regulation that have not yet been identified. Additionally, in this system, USP28 only targets Claspin but not PLK1 (both of which are subject to ubiquitination by the same E3 ligase). This raises another question-while deubiquitinase activity of USP28 is required to revert ubiquitination-mediated protein turnover, how it discriminates the different substrates remains elusive. Answers to this question can promote our understanding of the pathophysiological implications of USP28-mediated 
regulation on genotoxicity and cell cycle progression, which may shed light on improving strategies for cancer therapy.

\section{Targeting USP28 for cancer therapy}

DUBs have long been targeted for cancer treatment. For instance, CYLD, a member of the USP class of DUBs, was identified as the causal gene for the rare familial conditions of cylindromatosis and trichoepithelioma, of which the symptom is the development of multiple skin tumors. Loss of function of CYLD tiptoes the transcriptional activity of the NF- $\mathrm{\kappa B}$ pathway to the active side and drives many oncogenic processes ${ }^{6-8,13,35-38}$. Therefore, targeting CYLD may have important significance in the treatment of multiple cancers in lung, liver, colon, multiple myeloma, and melanoma ${ }^{39-42}$. Besides CYLD, additional DUBs, including A20, OTUD7B, USP11, H2A, BAP1, USP8, OTUD6B, UCH37, VCPIP1, USP7, COPS5, USP6, USP2, and USP15 have been implicated as potential targets in various cancers ${ }^{43-54}$. The clinical implications of dysregulated USP28 have been documented for various cancers as well. As a result, the significance of targeting USP28 for cancer treatment has been drawn more attention than before. Below, we will review the advances in USP28-targeted cancer therapy (Table 2).

\section{Targeting USP28 in non-small cell lung cancer (NSCLC)}

USP28 has been suggested as a therapeutic target for NSCLC. In one study, USP28 was found to be upregulated in the NSCLC tumors. Importantly, poor survivals were correlated with high levels of USP28 in patients. The cancer-promoting role of USP28 was confirmed by in vitro assays where overexpressing USP28 could enhance NSCLC cell proliferation while downregulating it induced apoptosis. Interestingly, USP28 may be targeted by a microRNA, miR4295, as USP28 3'UTR-driven luciferase activity was significantly reduced by miR- $4295^{55}$.

In a different study, however, miR-3940-5p was identified to target USP28 and CCND1 (Cyclin D1) in NSCLC. miR-3940-5p level was lower in NSCLC tumors than in matched adjacent normal tissues, and correlated with better clinicopathological features. Coincidently, USP28 was upregulated in NSCLC tumors and associated with poor prognosis of NSCLC patients. Therefore, miR-39405p may target CCND1 and USP28 to inhibit NSCLC growth. Indeed, downregulation of miR-3940-5p was concomitant with upregulation of CCND1 and USP28 in NSCLC tissues and cell lines. Importantly, miR-3940-5p suppressed proliferation and promoted apoptosis in NSCLC cells. It was verified that CCND1 and USP28 were direct targets of miR-3940-5p, and the phenotypes of NSCLC cell proliferation and apoptosis imposed by miR3940-5p could be attenuated by overexpression of CCND1 or USP28. Accordingly, the therapeutic value of targeting USP28 was further demonstrated by in vivo experiments where overexpressing miR-3940-5p inhibited the growth of NSCLC tumors. Therefore, USP28 and CCND1 may be promising diagnostic markers and therapeutic targets for NSCLC ${ }^{56}$.

\section{Targeting USP28 in breast cancer}

One study has revealed a critical mechanism underlying the epigenetic regulation by USP2 8 in breast cancer. An unbiased siRNA screening against all the human DUBs yielded USP28 as a bona fide deubiquitinase of LSD1 (lysine-specific demethylase 1). USP28 can interact with and stabilize LSD1. In clinical tumor samples, USP28 overexpression is correlated with LSD1 upregulation. Interestingly, LSD1 is a critical epigenetic regulator and controls pluripotency and differentiation by demethylating H3K4me1/2. Disrupting USP28 leads to LSD1 destabilization, which suppresses cancer stem cell-like characteristics in vitro and inhibits tumorigenicity in vivo. Therefore, targeting USP28 provides another treatment approach against breast cancer ${ }^{23}$.

In a follow-up study, it was further confirmed that USP28 could modulate epigenetic events in breast cancer. Both LSD1 and histone deacetylases (HDACs) facilitate breast cancer proliferation, and interestingly, HDAC5 could promote the stability of USP28. Overexpression of USP28 largely reversed HDAC5-knockdown-induced LSD1 protein degradation, suggesting HDAC5 positively regulates LSD1 by stabilizing USP28. Therefore, targeting USP28 may tune down the HDAC5/LSD1-mediated epigenetic process that drives breast cancer development and progression $^{57}$.

\section{Targeting USP28 in intestinal cancer}

As stated previously, USP28 can stabilize FBW7targeted proteins, exhibiting oncogenic activities. Importantly, USP28 is highly expressed in colon cancers, making it a potential pharmacological target to control cMYC-driving tumor cells. In a mouse model of colorectal cancer, in contrast to the FBW7-deletion phenotype, intestine-specific deletion of USP28 reduced intestinal tumors. Importantly, in mice carrying the tumors, USP28 deletion reduced tumor size and prolonged lifespan ${ }^{18}$. Interestingly, USP28 can also antagonize the ubiquitindependent degradation of two additional oncogenic proteins, c-Jun and Notch1, expanding the substrate list that is shared by both USP28 and FBW7. The therapeutic benefit of USP28 in colon cancer was further demonstrated in another study, where USP28 knockout could increase the life expectancy of animals harboring FBW7deficient colon tumors. Consistently, FBW7 substrates, including c-MYC, c-JUN, NICD1, and cyclin E1, which were upregulated in FBW7-deficient mice, were downregulated in the double-knockout mouse ${ }^{2}$. 


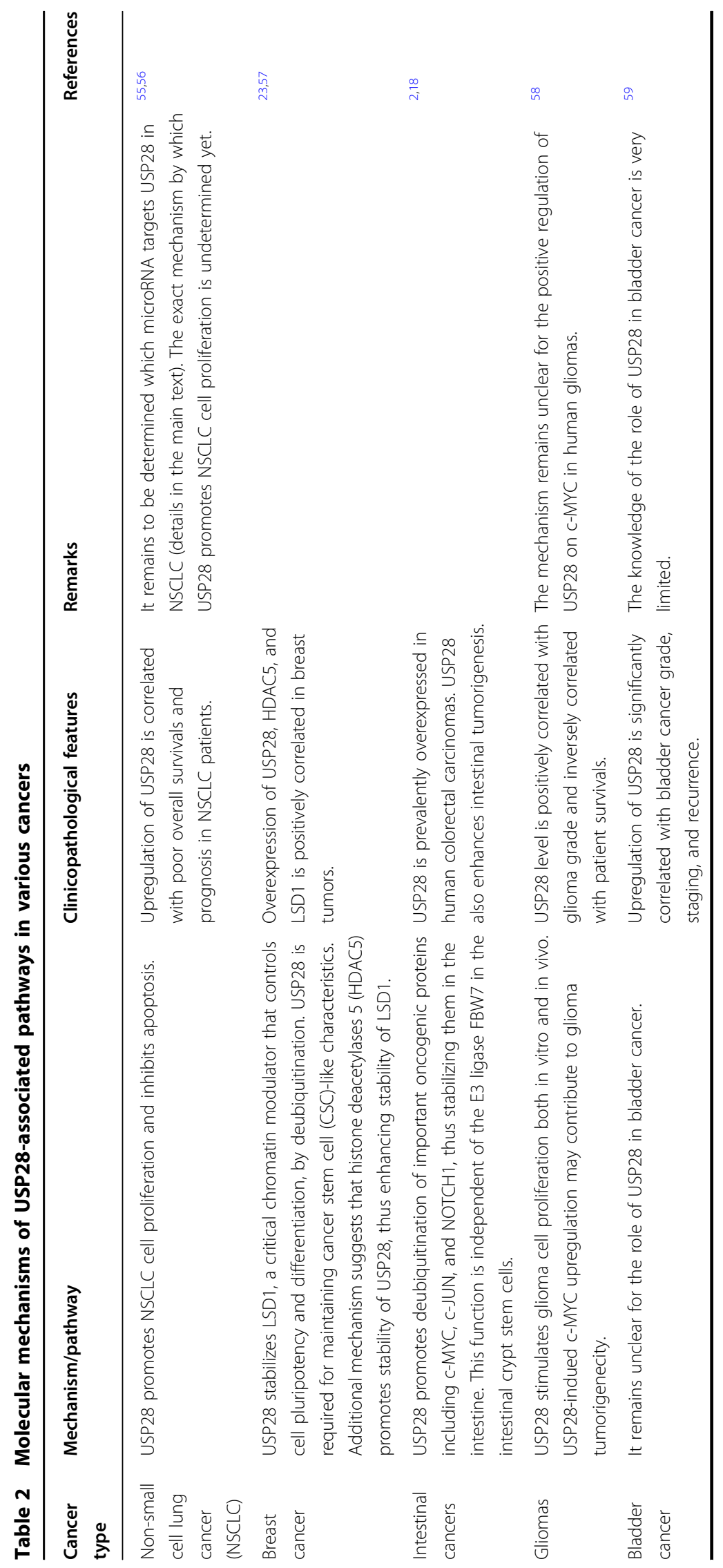




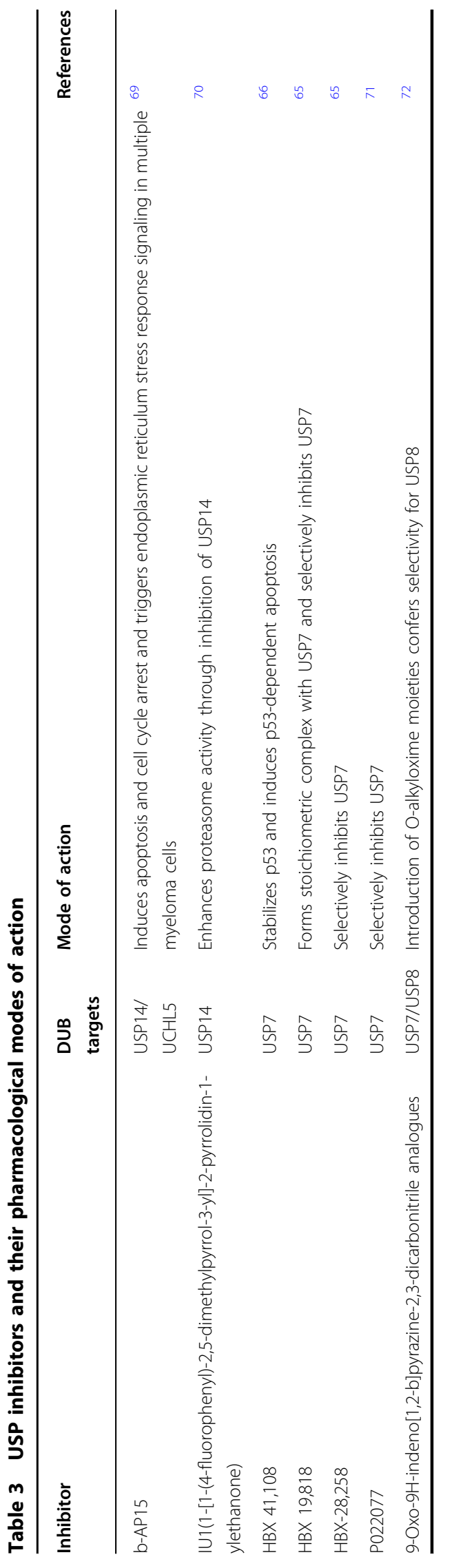

\section{Targeting USP28 in gliomas}

The significance of USP28 in glioma tumorigenesis has been demonstrated by its positive regulation on MYC, which is dysregulated in the majority of gliomas and difficult to target directly. It was determined that USP28 was upregulated in human gliomas but not in normal brain tissues. The USP28 protein level in human glioma tissues was directly correlated with glioma grade, and was inversely correlated with patient survivals. Ectopic USP28 expression promoted proliferation of SW1783 glioma cells both in vitro and in vivo, and conferred enhanced tumorigenicity in a nude mouse model. Disruption of USP28 in glioblastoma U373 cells suppressed anchorageindependent growth in vitro and tumorigenicity in vivo. Importantly, USP28 promoted MYC expression, which was required for USP28-induced cell growth in human glioma. Therefore, USP28 could be a new target of therapy for human malignant gliomas ${ }^{58}$.

\section{Targeting USP28 in bladder cancer}

The clinical significance of USP28 was also demonstrated in human bladder cancer. It has been determined that USP28 was overexpressed in bladder cancers compared to adjacent non-cancerous tissues at both the mRNA and protein levels. IHC examination confirmed that a majority of cancerous tissues displayed high USP28 levels. Furthermore, USP28 was strongly correlated with histopathological grade, clinical stage, tumor number, and recurrence rate. Interestingly, USP28 expression level can serve as an independent predictor of survival. Therefore, USP28 can potentially be valuable in the prognostic evaluation of bladder cancer ${ }^{59}$.

\section{Pharmacological studies for DUBs}

To date, pharmacological studies of DUBs have been focused on screening and identifying novel DUB inhibitors or antagonists for clinical regiment use. High throughput screenings in chemical libraries followed by structural-activity relationship studies have yielded fruitful compounds exhibiting selectivity for particular DUBs ${ }^{60-63}$. Due to the important role of USP7 in stabilizing p53, small molecules selective for USP7 have been a focus of the pharmacological studies for DUBs ${ }^{64-67}$. HBX 41108 was discovered to inhibit USP7 activity at a submicromolar range. Treatment with this compound leads to stabilization of p53 and subsequent repression of cancer cell growth ${ }^{66}$. A dual inhibitor selective for USP7 and USP47 was also reported to promote p53-induced apoptosis in cancer cells. Its efficacy was documented for a mouse xenograft model of myeloma and B-cell leukemia ${ }^{64}$. In Table 3 , we list some major USP inhibitors ${ }^{68-73}$. However, despite the advances in DUB-targeting drug discovery, the identified compounds are still in the early stage awaiting clinical approvals. To date, no compounds targeting USP28 have been reported yet, and no DUB-targeted drugs have yet been approved for 
clinical use. Structural studies were performed for only a handful of DUBs. Therefore, the challenges will offer new opportunities for the drug discovery of clinically relevant DUBs, including USP28.

\section{Concluding remarks}

Ubiquitination is one of the important post-translational modifications observed in the cell and plays multifaceted roles in cellular pathways, affecting protein turnover, signaling, and response to various stresses. DUBs, by removing the ubiquitin tags, have been shown to regulate stability of signaling proteins, which in turn affect the outputs of a variety of signaling cascades controlling cell proliferation, survival, DNA damage response, carcinogenesis, and metastasis. Several DUBs have been targeted for therapeutic applications, as exemplified by some promising drug discovery studies. To date, major roles of USP28 are demonstrated by its regulations of DDR and ubiquitinationdependent proteolysis pathways. It is likely that the functions of USP28 are cell/tissue type-dependent, and in a particular system, a specific aspect of these functions can be dominant. Regardless, USP2 8 may be a potentially beneficial therapeutic target for cancer treatment. Hence, its clinical relatedness and applications should be extensively explored, investigated, and evaluated.

\section{Acknowledgements}

This work was supported by grants from National Natural Science Foundation of China (No. 81072164, 81372430 and 81402525) and the Program of Shanghai Subject Chief Scientist (No.14XD1401200).

\section{Author details \\ 'Department of Radiation Oncology, Fudan University Shanghai Cancer Center, Shanghai 200032, China. ${ }^{2}$ Department of Oncology, Shanghai Medical College, Fudan University, Shanghai 200032, China. ${ }^{3}$ Department of Head and Neck Surgery, The University of Texas MD Anderson Cancer Center, Houston, Texas 77030, USA. ${ }^{4}$ Department of Breast Surgery, Fudan University Shanghai Cancer Center, Shanghai 200032, China. ${ }^{5}$ Department of Oncology, The First Hospital of Nanjing, Nanjing 210000, China. ${ }^{6}$ Cancer Institute, Longhua Hospital, Shanghai University of Traditional Chinese Medicine, Shanghai 200032, China}

\section{Conflict of interest}

The authors declare that they have no conflict of interest.

\section{Publisher's note}

Springer Nature remains neutral with regard to jurisdictional claims in published maps and institutional affiliations.

Received: 21 July 2017 Revised: 6 December 2017 Accepted: 7 December 2017

Published online: 07 February 2018

\footnotetext{
References

1. Zhen, Y., Knobel, P. A., Stracker, T. H. \& Reverter, D. Regulation of USP28 deubiquitinating activity by SUMO conjugation. J. Biol. Chem. 289, 34838-34850 (2014).

2. Diefenbacher, M. E. et al. Usp28 counteracts Fbw7 in intestinal homeostasis and cancer. Cancer Res. 75, 1181-1186 (2015).
}

3. Nijman, S. M. et al. A genomic and functional inventory of deubiquitinating enzymes. Cell 123, 773-786 (2005).

4. Fraile, J. M., Quesada, V., Rodriguez, D., Freije, J. M. \& Lopez-Otin, C. Deubiquitinases in cancer: new functions and therapeutic options. Oncogene 31, 2373-2388 (2012).

5. Komander, D., Clague, M. J. \& Urbe, S. Breaking the chains: structure and function of the deubiquitinases. Nat. Rev. Mol. Cell. Biol. 10, 550-563 (2009).

6. Brummelkamp, T. R., Nijman, S. M., Dirac, A. M. \& Bernards, R. Loss of the cylindromatosis tumour suppressor inhibits apoptosis by activating NFkappaB. Nature 424, 797-801 (2003).

7. Trompouki, E. et al. CYLD is a deubiquitinating enzyme that negatively regulates NF-kappaB activation by TNFR family members. Nature 424, 793-796 (2003).

8. Kovalenko, A. et al. The tumour suppressor CYLD negatively regulates NFkappaB signalling by deubiquitination. Nature 424, 801-805 (2003).

9. Valero, R. et al. Characterization of alternatively spliced products and tissuespecific isoforms of USP28 and USP25. Genome Biol. 2, RESEARCH0043 (2001).

10. Awakumov, G. V. et al. Amino-terminal dimerization, NRDP1-rhodanese interaction, and inhibited catalytic domain conformation of the ubiquitinspecific protease 8 (USP8). J. Biol. Chem. 281, 38061-38070 (2006).

11. $\mathrm{Hu}, \mathrm{M}$. et al. Crystal structure of a UBP-family deubiquitinating enzyme in isolation and in complex with ubiquitin aldehyde. Cell 111, 1041-1054 (2002).

12. $\mathrm{Hu}, \mathrm{M}$. et al. Structure and mechanisms of the proteasome-associated deubiquitinating enzyme USP14. Embo. J. 24, 3747-3756 (2005).

13. Komander, D. et al. The structure of the CYLD USP domain explains its specificity for Lys63-linked polyubiquitin and reveals a B box module. Mol. Cell. 29, 451-464 (2008).

14. Renatus, M. et al. Structural basis of ubiquitin recognition by the deubiquitinating protease USP2. Structure 14, 1293-1302 (2006).

15. Ye, Y. et al. Polyubiquitin binding and cross-reactivity in the USP domain deubiquitinase USP21. Embo. Rep. 12, 350-357 (2011).

16. Popov, N. et al. The ubiquitin-specific protease USP28 is required for MYC stability. Nat. Cell. Biol. 9, 765-774 (2007).

17. Welcker, M. \& Clurman, B. E. FBW7 ubiquitin ligase: a tumour suppressor at the crossroads of cell division, growth and differentiation. Nat. Rev. Cancer 8, 83-93 (2008).

18. Diefenbacher, M. E. et al. The deubiquitinase USP28 controls intestinal homeostasis and promotes colorectal cancer. J. Clin. Invest. 124, 3407-3418 (2014).

19. Schulein-Volk, C. et al. Dual regulation of Fbw7 function and oncogenic transformation by Usp28. Cell Rep. 9, 1099-1109 (2014).

20. Taranets, L., Zhu, J., Xu, W. \& Popov, N. Fbw7 and Usp28 - enemies and allies. Mol. Cell Oncol. 2, e995041 (2015).

21. Bohgaki, M. et al. The E3 ligase PIRH2 polyubiquitylates $\mathrm{CHK} 2$ and regulates its turnover. Cell. Death. Differ. 20, 812-822 (2013).

22. Zhang, D., Zaugg, K. Mak, T. W. \& Elledge, S. J. A role for the deubiquitinating enzyme USP28 in control of the DNA-damage response. Cell 126, 529-542 (2006).

23. Wu, Y. et al. The deubiquitinase USP28 stabilizes LSD1 and confers stem-celllike traits to breast cancer cells. Cell Rep. 5, 224-236 (2013).

24. Flugel, D., Gorlach, A. \& Kietzmann, T. GSK-3beta regulates cell growth, migration, and angiogenesis via Fbw7 and USP28-dependent degradation of HIF-1alpha. Blood 119, 1292-1301 (2012).

25. Ahmad, S. S., Duke, S., Jena, R., Williams, M. V. \& Burnet, N. G. Advances in radiotherapy. BMJ 345, e7765 (2012).

26. Bentzen, S. M. et al. Towards evidence-based guidelines for radiotherapy infrastructure and staffing needs in Europe: the ESTRO QUARTS project. Radiother. Oncol. 75, 355-365 (2005).

27. Delaney, G., Jacob, S., Featherstone, C. \& Barton, M. The role of radiotherapy in cancer treatment: estimating optimal utilization from a review of evidencebased clinical guidelines. Cancer 104, 1129-1137 (2005).

28. Wang, B., Matsuoka, S., Carpenter, P. B. \& Elledge, S. J. 53BP1, a mediator of the DNA damage checkpoint. Science 298, 1435-1438 (2002).

29. Lin, S. Y., Li, K., Stewart, G. S. \& Elledge, S. J. Human Claspin works with BRCA1 to both positively and negatively regulate cell proliferation. Proc. Natl. Acad. Sci. USA 101, 6484-6489 (2004).

30. Bassermann, F. et al. The Cdc14B-Cdh1-PIk1 axis controls the G2 DNAdamage-response checkpoint. Cell 134, 256-267 (2008).

31. Harper, J. W. \& Elledge, S. J. The DNA damage response: ten years after. Mol. Cell. 28, 739-745 (2007). 
32. Mailand, N., Bekker-Jensen, S., Bartek, J. \& Lukas, J. Destruction of Claspin by SCFbetaTrCP restrains Chk1 activation and facilitates recovery from genotoxic stress. Mol. Cell. 23, 307-318 (2006).

33. Mamely, I. et al. Polo-like kinase-1 controls proteasome-dependent degradation of Claspin during checkpoint recovery. Curr. Biol. 16, 1950-1955 (2006).

34. Peschiaroli, A. et al. SCFbetaTrCP-mediated degradation of Claspin regulates recovery from the DNA replication checkpoint response. Mol. Cell. 23, 319-329 (2006).

35. Biggs, P. J. et al. Familial cylindromatosis (turban tumour syndrome) gene localised to chromosome 16q12-q13: evidence for its role as a tumour suppressor gene. Nat. Genet. 11, 441-443 (1995).

36. Blake, P. W. \& Toro, J. R. Update of cylindromatosis gene (CYLD) mutations in Brooke-Spiegler syndrome: novel insights into the role of deubiquitination in cell signaling. Hum. Mutat. 30, 1025-1036 (2009).

37. Courtois, G. Tumor suppressor CYLD: negative regulation of NF-kappaB signaling and more. Cell. Mol. Life. Sci. 65, 1123-1132 (2008).

38. Massoumi, R. \& Paus, R. Cylindromatosis and the CYLD gene: new lessons on the molecular principles of epithelial growth control. Bioessays 29, 1203-1214 (2007).

39. Hellerbrand, $C$. et al. Reduced expression of CYLD in human colon and hepatocellular carcinomas. Carcinogenesis 28, 21-27 (2007).

40. Jenner, M. W. et al. Gene mapping and expression analysis of $16 \mathrm{q}$ loss of heterozygosity identifies WWOX and CYLD as being important in determining clinical outcome in multiple myeloma. Blood 110, 3291-3300 (2007).

41. Massoumi, R. et al. Down-regulation of CYLD expression by Snail promotes tumor progression in malignant melanoma. J. Exp. Med. 206, 221-232 (2009).

42. Zhong, S., Fields, C. R., Su, N., Pan, Y. X. \& Robertson, K. D. Pharmacologic inhibition of epigenetic modifications, coupled with gene expression profiling, reveals novel targets of aberrant DNA methylation and histone deacetylation in lung cancer. Oncogene 26, 2621-2634 (2007).

43. Enesa, K. et al. NF-kappaB suppression by the deubiquitinating enzyme Cezanne: a novel negative feedback loop in pro-inflammatory signaling. J. Biol. Chem. 283, 7036-7045 (2008).

44. Hussain, S., Zhang, Y. \& Galardy, P. J. DUBs and cancer: the role of deubiquitinating enzymes as oncogenes, non-oncogenes and tumor suppressors. Cell Cycle 8, 1688-1697 (2009).

45. Kato, M. et al. Frequent inactivation of A20 in B-cell lymphomas. Nature $\mathbf{4 5 9}$ 712-716 (2009).

46. LaFave, L. M. et al. Loss of BAP1 function leads to EZH2-dependent transformation. Nat. Med. 21, 1344-1349 (2015)

47. Luise, C. et al. An atlas of altered expression of deubiquitinating enzymes in human cancer. PLoS. ONE. 6, e15891 (2011).

48. Oliveira, A. M. et al. USP6 and CDH11 oncogenes identify the neoplastic cell in primary aneurysmal bone cysts and are absent in so-called secondary aneurysmal bone cysts. Am. J. Pathol. 165, 1773-1780 (2004).

49. Reincke, M. et al. Mutations in the deubiquitinase gene USP8 cause Cushing's disease. Nat. Genet. 47, 31-38 (2015).

50. Schmitz, R. et al. TNFAIP3 (A20) is a tumor suppressor gene in Hodgkin lymphoma and primary mediastinal B cell lymphoma. J. Exp. Med. 206, 981-989 (2009).

51. Shembade, N. et al. The E3 ligase Itch negatively regulates inflammatory signaling pathways by controlling the function of the ubiquitin-editing enzyme A20. Nat. Immunol. 9, 254-262 (2008).

52. Sun, S. C. Deubiquitylation and regulation of the immune response. Nat. Rev. Immunol. 8, 501-511 (2008).
53. Sun, W. et al. USP11 negatively regulates TNFalpha-induced NF-kappaB activation by targeting on IkappaBalpha. Cell. Signal. 22, 386-394 (2010).

54. Zhou, Q. et al. Loss-of-function mutations in TNFAIP3 leading to A20 haploinsufficiency cause an early-onset autoinflammatory disease. Nat. Genet. 48 67-73 (2016).

55. Zhang, L. et al. Overexpression of deubiquitinating enzyme USP28 promoted non-small cell lung cancer growth. J. Cell. Mol. Med. 19, 799-805 (2015).

56. Ren, K., Li, Y., Lu, H., Li, Z. \& Han, X. miR-3940-5p functions as a tumor suppressor in non-small cell lung cancer cells by targeting Cyclin D1 and ubiquitin specific peptidase-28. Transl. Oncol. 10, 80-89 (2017).

57. Cao, C. et al. Functional interaction of histone deacetylase 5 (HDAC5) and lysine-specific demethylase 1 (LSD1) promotes breast cancer progression. Oncogene 36, 133-145 (2017).

58. Wang, Z., Song, Q., Xue, J., Zhao, Y. \& Qin, S. Ubiquitin-specific protease 28 is overexpressed in human glioblastomas and contributes to glioma tumorigenicity by regulating MYC expression. Exp. Biol. Med. 241, 255-264 (2016).

59. Guo, G., Xu, Y., Gong, M., Cao, Y. \& An, R. USP28 is a potential prognostic marker for bladder cancer. Tumour Biol. 35, 4017-4022 (2014).

60. Ratia, K. et al. A noncovalent class of papain-like protease/deubiquitinase inhibitors blocks SARS virus replication. Proc. Natl. Acad. Sci. USA 105 16119-16124 (2008)

61. Mermerian, A. H., Case, A., Stein, R. L. \& Cuny, G. D. Structure-activity relationship, kinetic mechanism, and selectivity for a new class of ubiquitin Cterminal hydrolase-L1 (UCH-L1) inhibitors. Bioorg. Med. Chem. Lett. 17, 3729-3732 (2007)

62. Ghosh, A. K. et al. Structure-based design, synthesis, and biological evaluation of a series of novel and reversible inhibitors for the severe acute respiratory syndrome-coronavirus papain-like protease. J. Med. Chem. 52, 5228-5240 (2009).

63. Daviet, L. \& Colland, F. Targeting ubiquitin specific proteases for drug discovery. Biochimie 90, 270-283 (2008).

64. Weinstock, J. et al. Selective dual inhibitors of the cancer-related deubiquitylating proteases USP7 and USP47. ACS Med Chem. Lett. 3, 789-792 (2012).

65. Reverdy, C. et al. Discovery of specific inhibitors of human USP7/HAUSP deubiquitinating enzyme. Chem. Biol. 19, 467-477 (2012)

66. Colland, F. et al. Small-molecule inhibitor of USP7/HAUSP ubiquitin protease stabilizes and activates p53 in cells. Mol. Cancer Ther. 8, 2286-2295 (2009).

67. Chauhan, D. et al. A small molecule inhibitor of ubiquitin-specific protease-7 induces apoptosis in multiple myeloma cells and overcomes bortezomib resistance. Cancer Cell. 22, 345-358 (2012).

68. He, M. et al. The emerging role of deubiquitinating enzymes in genomic integrity, diseases, and therapeutics. Cell Biosci. 6, 62 (2016).

69. Tian, Z. et al. A novel small molecule inhibitor of deubiquitylating enzyme USP14 and UCHL5 induces apoptosis in multiple myeloma and overcomes bortezomib resistance. Blood 123, 706-716 (2014).

70. Lee, B. H. et al. Enhancement of proteasome activity by a small-molecule inhibitor of USP14. Nature 467, 179-184 (2010).

71. Tian, $X$. et al. Characterization of selective ubiquitin and ubiquitin-like protease inhibitors using a fluorescence-based multiplex assay format. Assay. Drug. Dev. Technol. 9, 165-173 (2011).

72. Colombo, M. et al. Synthesis and biological evaluation of 9-oxo-9H-indeno[1,2 b]pyrazine-2,3-dicarbonitrile analogues as potential inhibitors of deubiquitinating enzymes. ChemMedChem 5, 552-558 (2010).

73. D'Arcy, P., Wang, X. \& Linder, S. Deubiquitinase inhibition as a cancer therapeutic strategy. Pharmacol. Ther. 147, 32-54 (2015). 